\title{
A new endovascular treatment of a recurrent giant proximal basilar aneurysm after coiling
}

Weijian Jiang

\begin{abstract}
Background: Treatment of giant basilar aneurysms is extremely challenging, especially recurrences after previous coiling.

Case presentation: A 20-year-old male was referred for a recurrent giant proximal basilar aneurysm 3 months after coiling, with headache, blurred vision, and brainstem compression symptoms. Angiography showed that the previously placed coils were compacted within the caudal portion of the $43 \mathrm{~mm} \times 31 \mathrm{~mm}$ aneurysm, with spontaneous occlusion of the right vertebral artery and absence of the posterior communicating arteries. The diameter of the aneurismal neck, the afferent and efferent arteries was $6 \mathrm{~mm}, 3.5 \mathrm{~mm}$ and $4.1 \mathrm{~mm}$, respectively. A balloon-expandable covered-stent of $3.5 \mathrm{~mm} \times 10 \mathrm{~mm}$ was selected, matching the above 3 measurements; and deployed precisely across the aneurismal neck, immediately creating the "sub-complete reconstruction with intentional endoleak distal to aneurismal neck". Subsequently, 2 self-expanding $4.5 \mathrm{~mm} \times 37 \mathrm{~mm}$ stents were telescopically implanted within the covered-stent to adjust the angulation of the proximal basilar artery. After the procedure, the patient's symptoms gradually disappeared. Catheter angiography at 18 days, 3 months and 15 months demonstrated complete aneurismal exclusion from the patent vertebrobasilar artery, with dramatic elimination of aneurismal volume and reconfiguration of the compacted coils. The patient was asymptomatic at 15-month follow-up.
\end{abstract}

Conclusions: Sub-complete reconstruction with intentional endoleak distal to aneurismal neck can be safely achieved after implantation of a covered-stent and conventional stents in the case of a recurrent post-coiling proximal giant basilar aneurysm, with complete vascular reconstruction subsequently.

Keywords: Giant aneurysm, Basilar artery, Recurrent aneurysm, Covered-stent, Endovascular treatment

\section{Background}

Giant basilar aneurysms with a fundus diameter of greater than $25 \mathrm{~mm}$ are rare intracranial lesions and associated with an extremely dismal natural history as a result of progressive brainstem compression, subarachnoid hemorrhage, or thromboembolism. This grim prognosis has been improved with advances in open and endovascular neurosurgery, such as direct microsurgical clipping of the aneurysmal neck, stent-assisted coiling of the aneurysm, parent artery ligation or coiling with or without bypass [1-6]. However, even with the development of these techniques, treatment of

Correspondence: lichen-morning@163.com

New Era Stroke Care and Research Institute, PLA Rocket Force General Hospitial, 18 Xinjiekouwai Street, Beijing 100088, China giant basilar aneurysms still carry high rates of mortality and morbidity [7].

Further exploration is imperative for optimal management of giant basilar aneurysms. Flow diversion, such as the Pipeline Embolization Device (Medtronic, Ireland), has an on-label indication for aneurismal occlusion in intracranial internal carotid aneurysms. However, the Pipeline is off-label for use in large and giant vertebrobasilar aneurysms $[8,9]$. Mortality has been reported between $0 / 7$ and $4 / 10$, or $4 / 7$ in small case series $[8,9]$. These deaths may be associated with delayed aneurismal rupture, occlusion of perforators, nonaneurismal intracranial hemorrhage, and worsening of mass effect $[8,9]$. Covered-stents have been successfully used in giant or wide-necked cavernous carotid aneurysms [10], but there have been few reports on its use in the treatment 
of vertebrobasilar aneurysms so far, in all probability due to concern about side branch and perforator occlusions and subsequent infarction. In one report, delayed exclusion of a giant vertebrobasilar junction fusiform aneurysm was achieved 4 months after placement of a covered stent over the aneurysmal inflow zone to partially exclude the aneurysm [10]. However, the partial exclusion concept is not generally accepted.

Theoretically, a sub-complete reconstruction with intentional endoleak distal to the aneurismal neck (SCRED) can be created by deploying a covered-stent across the aneurismal neck with complete arterial wall apposition at the proximal end and deliberate incomplete apposition at the distal end. This approach may hemodynamically exclude the aneurysm, while preserving parent artery. The distal endoleak reverses blood flow between covered-stent and the arterial wall, and may supply side branch vessels arising from the parent artery as well as the aneurysm. Applying this novel concept, we successfully managed this difficult case of a giant recurrent proximal basilar trunk aneurysm.

\section{Case presentation}

History and examination

A 20-year-old male patient was referred to our institute, for the treatment of a recurrent giant aneurysm of the proximal basilar trunk 3 months after coiling at another hospital. On admission, the patient complained of headache, blurred vision, numbness of the right-side of the face and left upper and lower extremities, as well as weakness of his upper and lower extremities for the past month. Neurological examination revealed no dysfunction but impaired superficial sensation on his right face and left upper and lower limbs. Catheter angiography showed no abnormality of the anterior circulation, but the posterior communicating arteries (PcoA) were absent. There had been interval spontaneous occlusion of intracranial V4 segment of the right vertebral artery, and a $43 \mathrm{~mm} \times 31 \mathrm{~mm}$ aneurysm of lower basilar trunk supplied by left vertebral artery, with a $15 \mathrm{~mm} \times 18 \mathrm{~mm}$ residual lumen and a $6 \mathrm{~mm}$ aneurismal neck. Diameter of the aneurismal afferent and efferent artery (relative to the aneurysm) was $3.5 \mathrm{~mm}$ and $4.1 \mathrm{~mm}$, respectively. A robust inflow jet of the aneurysm was also noted, which was directed to the caudal right aspect of the aneurysmal sac so that the previously packed coils were compacted within the caudal portion of the aneurysm (Fig. 1).

\section{Multidisciplinary decision}

The main clinical and angiographic characteristics of the young patient were summarized as follows: (1) brainstem compression; (2) robust hemodynamic jet into the aneurysm with rapid recurrence and aneurysmal expansion within 3 months of coil embolization; (3) broad aneurismal neck of $>4 \mathrm{~mm}$; and (4) absence of good

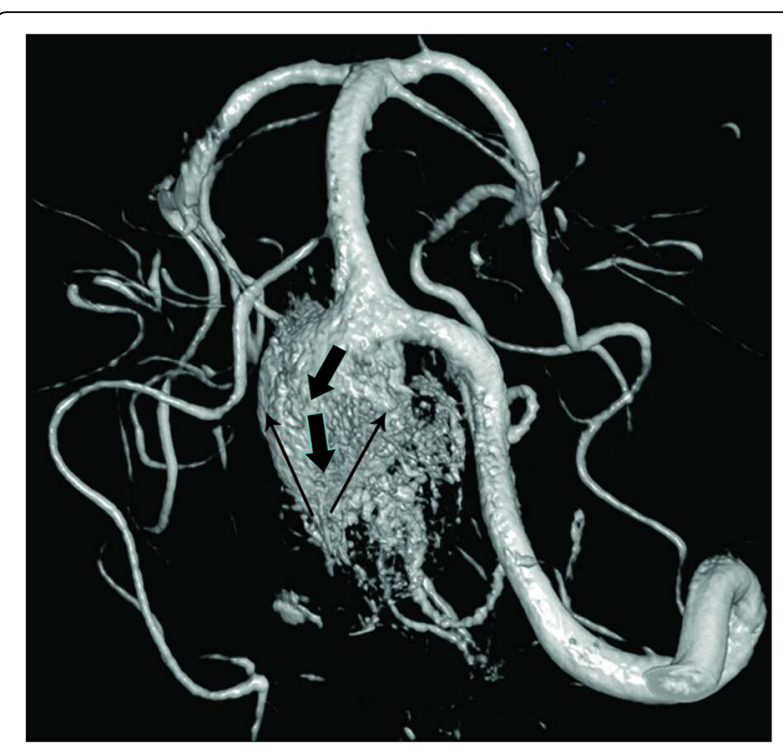

Fig. 1 Left vertebral angiography demonstrated a $43 \mathrm{~mm} \times 31 \mathrm{~mm}$ aneurysm of lower basilar trunk directed to caudally and to the right, with a $15 \mathrm{~mm} \times 18 \mathrm{~mm}$ residual lumen and a $6 \mathrm{~mm}$ aneurismal neck. Diameter of the afferent and efferent artery was $3.5 \mathrm{~mm}$ and $4.1 \mathrm{~mm}$, respectively. Robust inflow vector into the aneurysm was also noted (thick arrows), which directed to the caudal right aspect of the aneurysmal sac so that previously packed coils were compacted within the caudal portion of the aneurysm. Bilateral common carotid artery angiography showed no abnormal findings and absence of posterior communicating arteries (not shown), and right vertebral angiography revealed an intracranial V4 segment occlusion (not shown)

anterior to posterior circulation collaterals from PcoAs. Therefore, the objectives for treatment of the lesion were to exclude the aneurysm while preserving the parent artery and relevant perforators, to relieve mass effect, prevent hemorrhage, and avoid ischemic perforator and large artery stroke.

Open neurosurgery was considered, such as direct aneurysm clipping via a far lateral approach, or superficial temporal artery (STA) to superior cerebellar (SCA) or posterior cerebral artery (PCA) bypass plus distal occlusion of basilar artery just below the SCA via a subtemporal approach [1-6]. However, these procedures are extremely challenging for our neurosurgeons to perform with perceived high procedural risk.

Re-embolization with detachable coils was also considered, but the presence of pre-treatment mass effect was alarming. Additional coil volume and probable aneurysm expansion was deemed to be a high risk to result in increased mass effect. Further coil compaction due to the robust inflow jet was a concern as well. Coil sacrifice of the left vertebral artery alone was considered, however, the absence of PcoAs and pre-existent right vertebral artery occlusion would almost certainly result in complete posterior circulation infarction. 
The Pipeline Embolization Device was not available at the treating institution. The remaining endovascular option was the use of a covered-stent with the Willis covered-stent (MicroPort, Shanghai, China). The Willis is a balloon-expandable polymer covered-stent with a nominating inflation pressure of 6 atm. A $3.5 \mathrm{~mm} \times 10 \mathrm{~mm}$ size is available, which matched with the diameters of the parent vessel and the aneurismal neck in creating the SCRED.

\section{Procedure and Periprocedural management}

Written informed consent for this procedure was obtained from the patient and his family member. The institutional ethics committee approved the study protocol. Good Clinical Practice guidelines were used in accordance with the Declaration of Helsinki, and patient privacy was strictly protected. Consent from the patient for publication of this case study was also obtained.

After taking $75 \mathrm{mg}$ clopidogrel and $300 \mathrm{mg}$ aspirin daily for 5 days, the patient underwent the endovascular reconstruction procedure. Nimodipine $(0.6 \mathrm{mg} / \mathrm{h})$ was initiated intravenously to prevent artery spasm starting two hours before the procedure and maintained throughout the procedure. Heparin (3000 units) bolus was administered intravenously, followed by continuous at 1000 units/h, to maintain an activated coagulation time of approximately $220 \mathrm{~s}$ throughout the procedure.

The procedure was performed in our hybrid operating room equipped with an Artis Zeego angiographic system (Siemens AG, Forchheim, Germany). Under general anesthesia, a 6-French Neuron guiding catheter (Penumbra Inc., Alameda, USA) was advanced into the left vertebral artery. After 3-Dimension angiography, a $300 \mathrm{~cm}$ Transend microwire (Boston scientific corporation, Heredia, Costa Rica) and a Prowler SELECT Plus microcatheter (Codman Neurovascular, Chihuahua, Mexico) were negotiated across the aneurismal neck and reach the P2 segment of the right posterior cerebral artery under roadmap guidance. The guiding catheter was then advanced into the distal basilar artery over the assembly. The microcatheter was withdrawn while leaving the microwire in situ. The $3.5 \mathrm{~mm} \times 10 \mathrm{~mm}$ Willis covered-stent was conveyed into the distal end of the guiding catheter over the microwire. After withdrawal of the guiding catheter from the basilar artery to the distal left vertebral artery, the Willis was unsheathed and adjusted to make sure that its proximal end was located at the afferent artery $2 \mathrm{~mm}$ below the proximal edge of the aneurismal neck, and its distal end at the efferent artery just about $2 \mathrm{~mm}$ over the distal edge of the neck. The Willis was then deployed by inflating the balloon to 6 atm. (Fig. 2a). The sub-complete aneurysm exclusion was immediately created, with an intentional endoleak at the distal end of the covered-stent, which reversed blood flow from the basilar artery distal to the Willis (Fig. 2b). To ensure complete artery wall apposition at the proximal portion of the Willis stent, the balloon was re-inflated to $8 \mathrm{~atm}$ after withdrawal of the balloon $(6 \mathrm{~mm})$ to the proximal portion of the stent. Angulation of the proximal basilar artery at the level of the intentional endoleak origin was also noted (Fig. 2b), which was likely to be unfavourable for blood flow redirection to the normal middle and distal basilar artery. Two $4.5 \mathrm{~mm} \times 37 \mathrm{~mm}$ Enterprise self-expanding stents (Cordis Neurovascular, Miami, FL, USA) were telescopically deployed within the Willis stent from the mid basilar artery to the left vertebral artery (Fig. 2c), which changed the angulation sufficiently in the operators' judgement (Fig. 2d).

Low molecular weight heparin (Dalteparin calcium $0.4 \mathrm{ml}$ twice daily [Pfizer Manufacturing Belgium NV) was administrated for the first 3 postoperative days. Dual antiplatelet agents $(75 \mathrm{mg}$ clopidogrel and $100 \mathrm{mg}$ aspirin daily) were scheduled for 24 months.

\section{Clinical and imaging follow-up}

There were no procedure-related complications or complications related to increasing mass effect. At discharge (20 days later), the patient's headache had disappeared, and the other symptoms had also improved. The patient was asymptomatic at the 15-month follow-up.

Catheter angiography at 18 days (Fig. 3a), 3 months (Fig. 3b), and 15 months (Fig. 4) demonstrated that the aneurysm was completely excluded from the preserved vertebrobasilar artery, with dramatic reconfiguration of the previously "compacted" coils. On follow up, the coil mass was dense and closely apposed to the stent complex indicating aneurysm shrinkage. MRI T1WI (Fig. 5a), T2WI (Fig. 5b), and SPACE T1WI before (Fig. 5c) and after contrast enhancement (Fig. 5d) at 3 months also showed complete intra-aneurysm thrombosis.

\section{Discussion and Conclusions \\ A glimpse of the current management of giant basilar aneurysms}

Giant aneurysms in the posterior circulation are aggressively managed because of high rupture risk of up to $50 \%$ at 5 years if left untreated. The aims of intervention are protection from hemorrhage, relief of mass effect and prevention of thromboembolism.

However, open neurosurgical approaches to giant basilar aneurysms are challenging due to the deep location, complex anatomy, and proximity to vital perforating arteries [1, 3, 4, 8-10]. Recurrent lesions post coil embolization may be especially challenging due to the presence of packed coils which may hinder tissue apposition and aneurysm exclusion during microsurgical clipping [6]. Risks of $10 \%$ mortality and $38 \%$ morbidity were reported in treating giant posterior circulation aneurysms by direct 

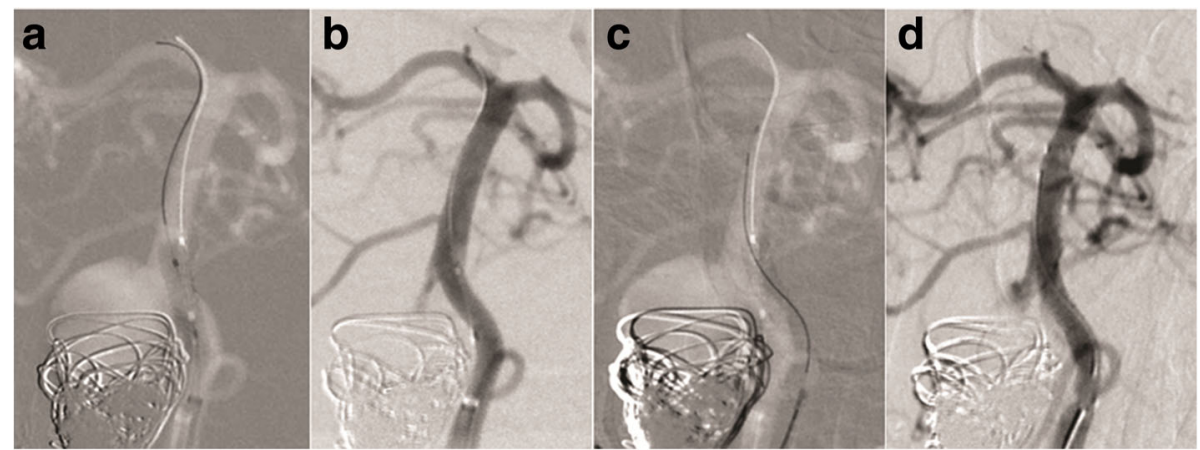

Fig. 2 A $3.5 \mathrm{~mm} \times 10 \mathrm{~mm}$ balloon-expandable Willis covered stent (MicroPort, Shanghai, China) to match the diameters of the aneurismal neck, afferent and efferent artery was precisely placed, so that its proximal end was located at the afferent artery $2 \mathrm{~mm}$ below the proximal edge of the aneurismal neck, and its distal end at the efferent artery just about $2 \mathrm{~mm}$ over the distal edge of the neck, and then deployed (a). A sub-complete aneurysm exclusion was immediately created, with an intentional endoleak at the distal end of the covered-stent, which reversed blood flow from the basilar artery distal to the Willis (b). An angulation of the proximal basilar artery at the level of the intentional endoleak origin was also noted (b), which was likely to be unfavourable for directing blood flow to the normal middle and distal basilar artery. Two $4.5 \mathrm{~mm} \times 37 \mathrm{~mm}$ Enterprise stents (Cordis Neurovascular, Miami, FL, USA) were coaxially deployed within the Willis from the middle basilar artery to the left vertebral artery (c), which changed the angulation (d)

neck clipping and parent artery clipping with or without bypass [7].

Compared with coiling smaller aneurysms, coiling giant aneurysms is very challenging for endovascular approaches due to a lower rate of complete aneurysm occlusion and higher recurrence rates despite use of stent assistance. In a study of 39 giant aneurysms in 38 patients with coiling, cumulative mortality at 30 days and at mean 25 months of follow-up was $16 \%$ and $29 \%$, respectively. The mortality was higher in posterior circulation aneurysms $(20 \%[2 / 10]$ and $60 \%,[6 / 10]$, respectively), and highest in basilar aneurysms (40\% [2/5] and $100 \%$ [5/5], respectively) [5].

Endovascular occlusion of the parent artery of giant vertebrobasilar aneurysms with or without bypass may be a better alternative for some selected patients. In a study of 13 giant vertebrobasilar aneurysms with good collaterals and tolerance to balloon test occlusion, parent artery coiling achieved a $92 \%$ rate of complete aneurysm occlusion. However, there still was one death and one recurrence during mean 28 months of follow-up [6]. Furthermore, parent vessel sacrifice without bypass is not suitable for patients with poor collaterals and failed balloon test occlusion.

The Pipeline Embolization Device has been approved in the treatment of large or giant wide-necked internal carotid aneurysms from the petrous to superior hypophyseal segments with extremely good outcomes, but the use of the device in vertebrobasilar aneurysms remains controversial due to uncertainty on safety $[8,9]$.

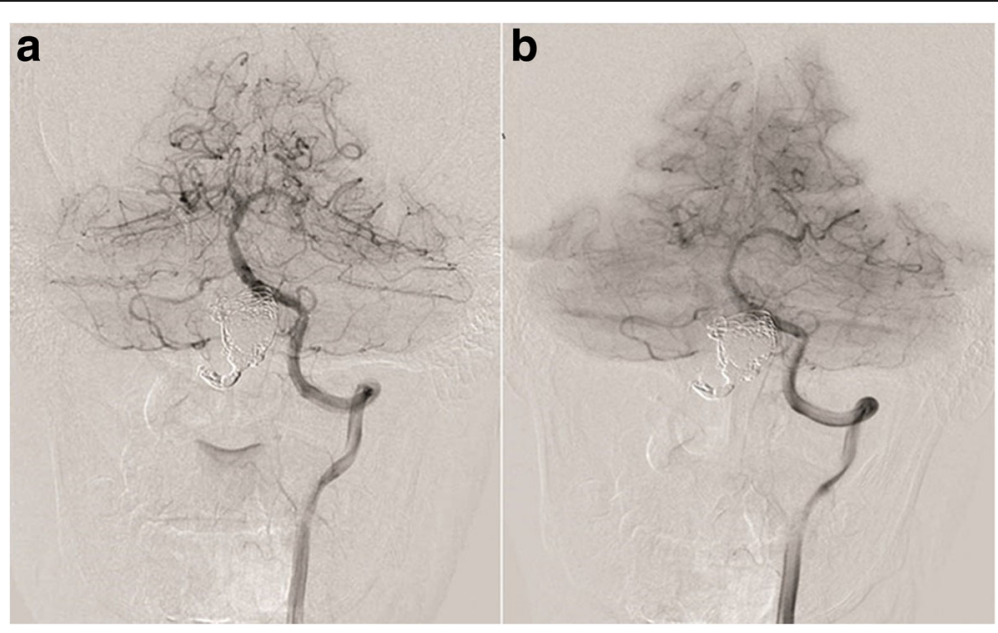

Fig. 3 Catheter angiography at 18 days (a) and 3 months (b) demonstrated that the aneurysm was completely excluded from the vertebrpbasilar artery 


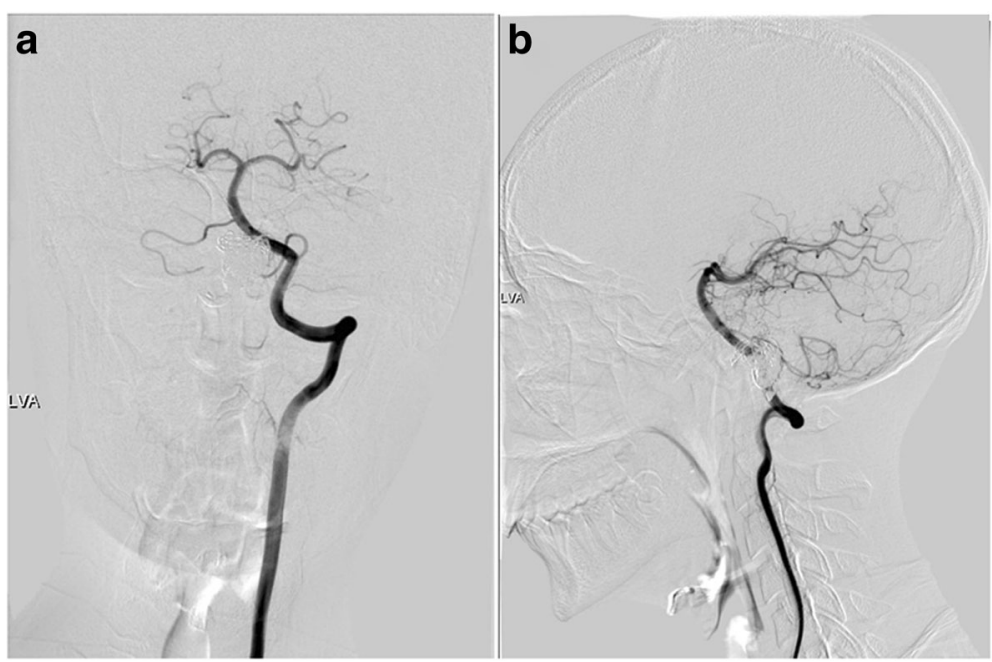

Fig. 4 Catheter angiography at 15 months (Fig. 4), both anteroposterior (a) and lateral (b) views were displayed. The aneurysm was completely excluded from the vertebrpbasilar artery and dense reconfiguration of the pre-existing coils suggesting aneurysm shrinkage

In 2002, Islak et al. proposed a partial exclusion concept with the use of covered-stent to manage a giant vertebrobasilar junction fusiform aneurysm [10]. In that case, a shorter covered-stent was navigated into a longer bare metal stent implanted across the aneurismal neck in advance, and deployed precisely covering the aneurismal inflow zone. The lesion was partially excluded immediately, and completely excluded at follow up 4 months later [10]. However, the concept is not generally accepted.

\section{Our experiences in the current case}

We modified the partial exclusion concept into one of sub-complete reconstruction with intentional endoleak distal to aneurismal neck, and successfully applied it in the current case of a symptomatic recurrent giant basilar aneurysm after coiling. The SCRED was safely and effectively created immediately, and resulted in complete vascular reconstruction at an early time point, with gradual disappearance of symptoms.

Two main observations are discussed as follows:

First, the SCRED was immediately achieved as the patient's efferent artery was larger than his afferent artery, which is a prerequisite of creating the reversed blood from the distal end of parent artery with the use of a balloon-expandable covered-stent; and the covered-stent used was well matched in size with the afferent artery, efferent artery and aneurismal neck. So, precisely measuring the 3 key diameters and choosing the appropriately sized covered-stent in advance are crucial when applying the SCRED concept to reconstruct vasculature. Additionally, the coaxial deployment of the Enterprise self-expanding stents inside the covered stent may have played an important role for early aneurysmal exclusion
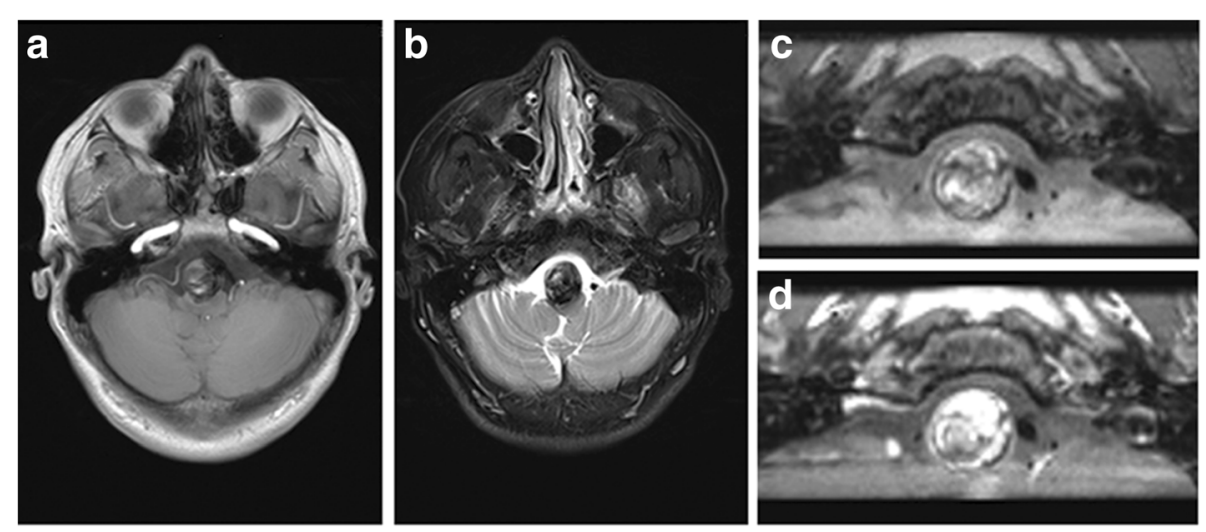

Fig. $5 \mathrm{MRI}$ T1WI (a), T2WI (b), and SPACE T1WI before (c) and after contract enhancement (d) at 3 months showed a complete intra-aneurysm thrombosis 
and complete vascular reconstruction, by decreasing the angulation of the proximal basilar artery, and directing the blood flow away from the aneurysm.

Second, the aim of protecting side branches and perforators was achieved as well, as intended. Covered-stent implantation can effectively exclude giant aneurysms from the patent arteries with no need of coil embolization, and in turn, may play a role in protection from delayed hemorrhage while relieving mass effect [10]. However, covered-stents may isolate perforators originating from the arterial segment covered by the covered-stent, and subsequently lead to perforator strokes and even fatal perforator strokes $[8,9]$. Perforating arteries are plentiful in the basilar artery, with a caudal group comprised of 2 to 5 perforators, a middle group with 5 to 9 perforators, and a rostral group with 1 to 5 perforators. Though the distal end of the covered stent was deployed in the caudal basilar artery, the arterial wall apposition was deliberately incomplete in this segment, so that our patient remained well at the follow-up of 15 months, suggesting that the reversed blood flow was sufficient to supply the caudal group perforators.

The outcome of our case was satisfactory, but further in depth studies are needed to realize the exact function and role of a "SCRED" and the durability in the treatment of giant basilar trunk aneurysms.

Sub-complete reconstruction with intentional endoleak distal to the aneurismal neck was safely achieved after implantation of covered-stent and conventional stents in the case of giant post-coil recurrent proximal basilar aneurysm, with subsequently complete vascular reconstruction. Evaluation of safety and efficacy, and indications for this technique require further study.

\footnotetext{
Abbreviations

PCA: Posterior cerebral artery; PcoA: Posterior communicating arteries; SCA: Superior cerebellar artery; SCRED: Sub-complete reconstruction with intentional endoleak distal to the aneurismal neck; STA: Superficial temporal artery
}

\section{Acknowledgements \\ Many people have offered me valuable help in this case writing, including my colleague, my students and my best friends. First, I would like to express my heartfelt gratitude to Dr. Xian Fu and Dr. Han-Chen Qiu. They had assisted me for this surgery, and offered many useful suggestions. Then my thanks would go to my best friend Ferdinand Hui and my student Dr. Chen Li for their earnest revise of the English language.}

\section{Funding}

This study was founded by National Key Basic Research Program of China (973 program) (grant No. 2013CB733805) and Program of National Natural Science Foundation of China (grant No.81070925 and 81,471,767) to Weijian Jiang.

\section{Availability of data and materials}

All data generated or analysed during this study are included in this published article.

\section{Author's contributions}

Weijian Jiang has performed this surgery, drafted and revised the manuscript.
Ethics approval and consent to participate

Written informed consent to participate in the study was obtained from the patient's guardian. The present study was approved by ethics committee of PLA Rocket Force General Hospital (2014-083).

\section{Consent for publication}

The patient has consented for publication of his case and the signed form is available with the author.

\section{Competing interests}

The authors declare that they have no competing interests.

Received: 3 January 2017 Accepted: 10 October 2017

Published online: 04 December 2017

References

1. Cantore G, Santoro A, Guidetti G, Delfinis CP, Colonnese C, et al. Surgical treatment of giant intracranial aneurysms: current viewpoint. [J] Neurosurgery. 2008;63:279.

2. Kalani MY, Ramey W, Albuquerque FC, McDougall CG, Nakaji P, et al. Revascularization and aneurysm surgery: techniques, indications, and outcomes in the endovascular era. [J]. Neurosurgery. 2014;74:482

3. Kalani MY, Zabramski JM, Nakaji P, Spetzler RF. Bypass and flow reduction for complex basilar and vertebrobasilar junction aneurysms []]. Neurosurgery. 2013;72:775-6.

4. Spetzler RF, Riina HA, Lemole GM, Jr. Giant aneurysms. [J]. Neurosurgery. 2001;49:902-8.

5. Jahromi BS, Mocco J, Bang JA, Gologorsky Y, Siddiqui AH, et al. Clinical and angiographic outcome after endovascular management of giant intracranial aneurysms [J]. Neurosurgery. 2008;63:662.

6. Lubicz B, Leclerc X, Gauvrit JY, Lejeune JP, Pruvo JP. Giant vertebrobasilar aneurysms: endovascular treatment and long-term follow-up. [J]. Neurosurgery. 2004;55:316-23.

7. Parkinson RJ, Eddleman CS, Batjer HH, Bendok BR. Giant intracranial aneurysms: endovascular challenges. []]. Neurosurgery. 2006;62:1336-45.

8. Meckel S, McAuliffe W, Fiorella D, Taschner CA, Phatouros C, et al. Endovascular treatment of complex aneurysms at the vertebrobasilar junction with flow-diverting stents: initial experience. [J]. Neurosurgery. 2013;73:386-94.

9. Siddiqui AH, Abla AA, Kan P, Dumont TM, Jahshan S, et al. Panacea or problem: flow diverters in the treatment of symptomatic large or giant fusiform vertebrobasilar aneurysms. [J] J Neurosurg. 2012;116:1258.

10. Islak C, Kocer N, Albayram S, Kizilkilic O, Uzma O, et al. Bare stent-graft technique: a new method of endoluminal vascular reconstruction for the treatment of giant and fusiform aneurysms. [J] AJNR Am J Neuroradiol. 2002;23:1589-95.

Submit your next manuscript to BioMed Central and we will help you at every step:

- We accept pre-submission inquiries

- Our selector tool helps you to find the most relevant journal

- We provide round the clock customer support

- Convenient online submission

- Thorough peer review

- Inclusion in PubMed and all major indexing services

- Maximum visibility for your research

Submit your manuscript at www.biomedcentral.com/submit
C) Biomed Central 